\title{
Clinical manifestations of hypothyroidism in residents of western region of Nepal: A case control study
}

\author{
Paudel B, ${ }^{1 *}$ Paudel $\mathrm{K},{ }^{1}$ Upadhaya $\mathrm{TL}^{1}$
}

${ }^{1}$ Dept. of Medicine, Gandaki Medical College Teaching Hospital, Pokhara, Nepal

\author{
*Corresponding Author: \\ Dr Badri Paudel, MD, \\ Associate Professor, Dept. of Medicine, \\ Gandaki Medical College Teaching Hospital, Pokhara, \\ Nepal \\ Email: mail@badripaudel.com
}

\section{Citation}

Paudel B, PaudelK, Upadhaya TL. Clinical manifestations of hypothyroidism in residents of western region of Nepal: A case control study. Nepal Journal of Medical Sciences 2013;2(1):62-5

\begin{abstract}
Background: This study was designed for the evaluation of the difference in the common signs and symptoms of hypothyroidism in our population from already available literature.

Methods: In this study we have compared the symptoms and signs of hypothyroid and euthyroid patients visiting to Gandaki Medical College Teaching Hospital (GMCTH) from April 2011 to October 2011. We compared the 18 common signs and symptoms of hypothyroidism in our patients and analyzed by SPSS software.

Results: Of the 2483 patients visiting to the GMCTH, 665 patients were included in the study and were examined. After the laboratory investigations, 98 were identified as the cases of hypothyroidism, the rest were declared as euthyroid and selected as controls. Lethargy, cold intolerance, constipation and paresthesia were the commonest symptom while facial oedema and bradycardia were the most prevalent sign in our population.
\end{abstract}

Conclusions: The most common signs and symptoms of hypothyroidism in the western region of Nepal (that is one of the iodine deficient areas in Nepal) were different from other studies. It seems that strong clinical suspicion on the basis of symptoms and signs elicited by physicians and laboratory confirmation are the only reliable methods for diagnosis of hypothyroidism.

Keywords: Hypothyroidism; common; signs; symptoms; case control studies

\section{Background:}

Eypothyroidism is a common endocrinological disorder. It is a clinical state resulting from an insufficient amount of circulating thyroid hormone to support normal body function. It may exist in utero or develop in infancy, childhood or even in adult life. It is characterized by a generalized reduction in metabolic function that most often manifests as a slowing of physical and mental activity. However, clinical features vary significantly among different populations owing to their climate, education status and awareness about the disease and also depends upon its cause, duration, and severity. The spectrum extends from sub-clinical to overt hypothyroidism to myxedema coma. ${ }^{1}$

Many of the common signs and symptoms of hypothyroidism can be found frequently also in euthyroid patients. Common symptoms such as fatigue, lethargy and constipation have 
limited diagnostic value, while weakness, insomnia and loss of memory are usually attributed to old age. ${ }^{2}$ The presentation of hypothyroidism is non-specific and high degree of suspicion is required for its early diagnosis. ${ }^{3}$ The demand for thyroid functions test over the past 20 years has increased due to the non-specific symptom of hypothyroidism. Unfortunately, there is very little information available on this subject from our part of the world. This study was designed to evaluate the clinical presentations of hypothyroidism in a population of iodine deficient area in western part of Nepal and then comparing it to the already available results from other parts of the world.

\section{Methods:}

A case control study was carried out at the Gandaki Medical College Teaching Hospital (GMCTH) Pokhara, Nepal. All the patients coming to GMCTH for their thyroid work up from April 2011 to October 2011 were interviewed and examined by a group of trained physicians with the help of a standard questionnaire, after obtaining a verbal consent. All patients with already established diagnosis of hypothyroidism, thyroid ablation or thyroidectomy, co-existing infections, thyroid or other malignancies, hyperthyroidism, sub-clinical hypothyroidism, pregnancy and those already on thyroid hormone replacement therapy were excluded from the study. There was no age limit specified for inclusion in the study. Both the doctors and the patients were unaware of the thyroid status of the patients at the time of interview.

The diagnosis of hypothyroidism was established after the availability of results of laboratory investigations on the basis of both clinical judgment and the laboratory investigations. The laboratory tests comprised of serum free triiodthyronine (FT3), free thyroxine (FT4) and thyroid stimulating hormones (TSH) by Enzyme-linked immunosorbent assay (ELISA) radioimmunoassay (Ranbaxy Kit, India). The questionnaire looked at the common symptoms and signs of hypothyroidism as mentioned in the literature.

The symptoms asked were dyspnoea, hoarseness, odynophagia, changes in bowel habits, temperature intolerance, lethargy, change in body weight, appetite, paresthesia, hair loss, body aches, depression, anxiety and menstrual abnormalities, mainly menorrhagia. The signs included pulse, deep tendon reflexes, skin changes like dry/ coarse skin and coarse hair, non-pitting peripheral oedema, and oedema of face.

The data were entered and analyzed using SPSS statistical software package, version 10.0 for Windows (SPSS. Inc, Chicago, IL). The results of the above mentioned variables were compared between hypothyroid and euthyroid patients using Chi Square or Fischer's exact test as appropriate.

\section{Results:}

Of the 2483 patients coming to the GMCTH, 665 patients were included in the study and were examined. After the laboratory investigations, 98 were identified as the cases of hypothyroidism, the rest were declared as euthyroid and selected as controls. The data analysis of the 98 hypothyroidism patients studied showed, 81 females and 17 males with a mean age of $42 \pm 13.4$ years (mean age for male Vs female: $52.8 \pm 18.2$ Vs $40.7 \pm 11.4) .28$ (28.6\%) patients had Hashimoto thyroiditis and $5(5.1 \%)$ cases had sub acute thyroiditis while remaining 65 (66.3\%) cases were idiopathic most probably iodine deficiency. The most common symptoms complained by our study population was lethargy; a total of 96 patients among the cases while 38 of the euthyroid control complained of it. Cold intolerance, constipation, paresthesia, weight gain were other common symptoms according to descending order of frequency (Table1). The most common sign present in hypothyroid patients were facial edema, pedal edema and bradycardia. The other symptoms complained by the study population and sign elicited by the physicians in both cases and controls are shown in Table 1.

Table-1: Frequency of the clinical features observed in hypothyroid and euthyroid patients.

\begin{tabular}{lccc}
\hline \multicolumn{1}{c}{ Symptoms } & $\begin{array}{c}\text { Hypothyroidism } \\
(\mathrm{N}=98)\end{array}$ & $\begin{array}{c}\text { Euthyroid } \\
(\mathrm{N}=567)\end{array}$ & P value \\
\hline Lethargy & 96 & 38 & 0.0001 \\
Facial Puffiness & 80 & 10 & 0.0001 \\
Cold intolerance & 80 & 20 & 0.0001 \\
Pedaledemanonpitting & 76 & 12 & 0.0001 \\
Constipation & 65 & 24 & 0.0001 \\
Paresthesia & 63 & 26 & 0.0001 \\
Weight gain & 43 & 12 & 0.0001 \\
Depression & 32 & 30 & 0.17 \\
Bradycardia & 29 & 10 & 0.0001 \\
Anxiety & 27 & 18 & 0.04 \\
Decreased appetite & 20 & 10 & 0.005 \\
Dry skin & 18 & 15 & 0.29 \\
Swelling in the neck & 15 & 8 & 0.04 \\
Hair fall & 16 & 8 & 0.01 \\
Insomnia & 10 & 12 & 0.15 \\
Menorragia & 3 & 1 & 0.10 \\
Weight loss & 2 & 2 & 0.09 \\
Vitligo & 1 & 0 & 0.14 \\
\hline
\end{tabular}




\section{Discussion:}

The clinical manifestation of chronic hypothyroidism may develop insidiously as compared to acute hypothyroidism, resulting from ablation or thyroiditis, associated with rapid onset of symptoms. The wide variability in the presentation further confounds the recognition of this condition. Among patients in general medicine practice presenting with the common complaints relating to hypothyroidism like constipation, weight gain, menstrual irregularities, fatigue, cold intolerance, depression and galactorrhea etc., only $4 \%$ are actually hypothyroid, and fewer than $2 \%$ have a TSH level at least $5 \mathrm{mU} / \mathrm{L}$ above normal. ${ }^{4}$ In contrast, more specific symptoms and signs include slow movement, coarse skin, decreased sweating, hoarseness, paresthesia, cold intolerance, periorbital oedema, and delayed deep tendon relaxation. ${ }^{5,6}$

In our study, we have found the female preponderance in hypothyroidism as expected. The female to male ratio in cases was 4.8:1 which is similar to what has been reported in the literature. The female to male ratio in control group was $6.5: 1$, making the results comparable with cases and decreasing any bias in clinical symptoms coming from different gender. In other studies the most sufferers were in the third and fourth decades of the life. ${ }^{7}$ But in our study it was seen in the lower decades of the life (second, third decade). May be more environmental antigen exposure and nutritional deficiency are among the probable causes suggested for this discrepancy.

Clinical features of hypothyroidism vary significantly among different populations owing to their climate, education status and awareness about the disease. The presentation of hypothyroidism is altered in the elderly in comparison with young patients; in that there are fewer signs or symptoms and diminished frequency of some classic signs. ${ }^{8}$

Lethargy, cold intolerance, constipation, paresthesia, weight gain, decreased appetite were the most common complaints in this study, which were significantly more prevalent than the control group $(\mathrm{P}<0.0001)$. Lethargy, cold intolerance, hair loss, difficulty concentration and poor memory, constipation, weight gain, poor appetite, dyspnea, hoarseness of voice, menorrhagia, paresthesia, impaired hearing are reported as the most common symptoms in other studies. Cold intolerance, due to the reduced basal metabolism and cardiac output; has been reported a very common symptom of hypothyroidism. ${ }^{7,9}$

The most specific and discriminating features of hypothyroidism are decreased sweating, hoarseness of voice, paraesthesias, cold intolerance, delayed reflexesand periorbital oedema which are significantly different than in our study.

The signs of hypothyroidism as elicited by the physicians were more specific and helpful in identification of hypothyroid patients (Table 1). These comprised facial oedema, non-pitting peripheral oedema, and bradycardia $(p<0.001)$. According to our study, menorrhagia, depression, dry / coarse skin and weight loss, are the symptoms that were not significantly different in hypo- and euthyroid patients $(p>0.05)$.

When we compare our study to already available data on this subject, we realize that most of the signs and symptoms have the same trend though the prevalence might be different. For example, weakness has been reported as one of the commonest symptoms of hypothyroidism with a prevalence of $98 \%$, and 99\% in Murray's and Lermann's series respectively and $25.5 \%$ in Watanakunakorn's study (Table-2). ${ }^{10-1310-13}$

Table-2: Comparison of symptoms and signs reported by previous studies

\begin{tabular}{|c|c|c|c|c|}
\hline & $\begin{array}{c}\text { Murray } \\
\text { series }\end{array}$ & Lermann & $\begin{array}{l}\text { Watana- } \\
\text { kunakorn }\end{array}$ & Khurram \\
\hline Sample size (n) & 100 & 77 & 400 & 106 \\
\hline Weakness & 98 & 99 & 26 & - \\
\hline Lethargy & 85 & 91 & 25 & 67.9 \\
\hline Constipation & 54 & 61 & 37 & 66.0 \\
\hline Body aches & 36 & - & 10 & 60.4 \\
\hline Depression & 60 & - & - & 53.7 \\
\hline Cold intolerance & 95 & $60-95$ & 58 & 50.9 \\
\hline Weight gain & 76 & $50-75$ & 48 & 44.9 \\
\hline Paresthesia & 56 & - & - & 46.2 \\
\hline Palpitations & 23 & 31 & - & - \\
\hline Menorrhagia & 33 & 32 & 16 & 38.6 \\
\hline Hoarseness & 74 & 52 & 48 & 36.7 \\
\hline Decreased appetite & 40 & - & 14 & 32.1 \\
\hline Dyspnea & 72 & 55 & 12 & 30.2 \\
\hline Odyno-phagia & - & 3 & 8 & 12.2 \\
\hline Nervousness/Anxiety & 51 & 35 & 13 & 12.2 \\
\hline Hair loss & 41 & 57 & 32 & 8.3 \\
\hline Edema of face & 95 & 79 & 67 & 63.3 \\
\hline Peripheral edema & 57 & 55 & - & 60.6 \\
\hline Skin changes & 70-79 & 97 & 89 & 14.7 \\
\hline $\begin{array}{l}\text { Delayedrelaxationof } \\
\text { deeptendonreflexes }\end{array}$ & - & - & 43 & 20.9 \\
\hline Exopthalmos & 11 & - & 1 & 1.8 \\
\hline
\end{tabular}

In our study too, lethargy was mentioned by $96 \%$ of the cases and was the most prevalent feature. Similarly, cold intolerance, that was found in $89 \%$ of the Lermann's 
series and $93 \%$ of the Murray's, was prevalent in about $80 \%$ of our cases, which is quite higher to the $58.25 \%$ in Watanakunakorn and $50.9 \%$ in Irfan M studies. ${ }^{10-13}$

While skin changes like dry, coarse skin, hoarseness and hair-loss were not mentioned as commonly in our study as they had been in other series However, we found that these symptoms were significantly specific for hypothyroidism, which was similar to what has been described in the literature. On the other hand, constipation, weight gain, oedema of the face and non-pitting peripheral oedema, had quite comparable prevalence to all the studies.

There had been reports that indicate that the prevalence of hypothyroidism increase in the elderly. ${ }^{14,15}$ In contrast to that, we had $27(27.5 \%)$ patients above fifty years of age in this hypothyroid group and $80(14.1 \%)$ in euthyroid control group. Though this study clearly shows an increasing trend of hypothyroidism with growing age, more data from different community based studies is required to call it statistically significant. The reason for the smaller sample size in the older age group could be that lesser number of elderly patients is brought to a diagnostic centre in our setting.

Most of the information that we get about the clinical manifestations of hypothyroidism is from literature of the western countries. Their level of education, awareness about the diseases, and health care facilities are quite different from ours. The climate and cultural norms are also very dissimilar. The pathophysiology of the disease always remains the same, but the perception of symptoms, their recognition and expression differ in every population as governed by the above mentioned determinants.

\section{Conclusions:}

The diagnosis of hypothyroidism may be overlooked for several years because of the insidious and non-specific nature of symptoms that further complicates the detection of hypothyroidism. Therefore, it is very critical to have a high degree of suspicion in assessing the patients having such a vague presentation. Hence, there is a need for every community to define the expression and prevalence of their common disorders in order to recognize them early and correctly; however, a support from laboratory investigations should be sought whenever and wherever required.

\section{Conflict of interest: none}

\section{References:}

1. Weetman AP. Hypothyroidism: screening and subclinical disease. BMJ 1997;314:1175-8.
2. Lindsay RS, Toft AD. Hypothyroidism. Lancet 1997;349:413-7.

3. Skinner GRB, Thomas R, Taylor M. Thyroxine should be tried in clinically hypothyroid but biochemically euthyroid patients. BMJ 1997;314:1764.

4. Schectman JM, Kallenberg GA, Shumacher RJ, et al. Yield of hypothyroidism in symptomatic primary care patients. Arch Intern Med 1989;149:861-4.

5. Billewicz WZ, Chapman RS, Crooks J, et al. Statistical methods applied to diagnosis of hypothyroidism. Q J Med 1969;38:255-66.

6. Utiger RD. Hypothyroidism. In: Degroot LJ eds. Endocrionolgy. $3^{\text {rd }}$ ed. Philadelphia, W.B. Sanders Company, 1995, pp 752-768.

7. Ebadi SAR, Afshar M. Epidemiology of hypothyroidism in Gilan. Kashan University of Medical Sciences and Health Services 2004;31:30-5.

8. Doucet J, Trivalle C, Chassagne P, et al. Does age play a role in clinical presentation of hypothyroidism? J Am Geriatr Soc 1995;43:592-3.

9. Sharifi H, Emami AH, Moosavi H, et al. Clinical and Para clinical criteria for Thyroid disorders in patients over 15 years. Feiz J Kashan University of Medical Sciences and Health Services 2001;19:40-3.

10. Lerman J, Means JH. The gastric secretion in exopthalmic goiter and myxoedema. J Clin Invest 1932;11:167.

11. Degroot LJ, Larsen PR, Hennemann G. Eds: Adult hypothyroidism. In: The thyroid and its diseases. $6^{\text {th }}$ ed. New York, Churchill Livingstone, 1996.pp 323-370.

12. Watanakunakorn C, Hodges RH, Evans TC. Myxedema: a study of 400 cases. Arch Intern Med 1965;116:183-90.

13. Khurram IM, Choudhry KS, Muhammad K, et al. Clinical presentation of hypothyroidism: a case control analysis. J Ayub Med Coll Abbottabad 2003;15:45-9.

14. Sawin CT, Castelli WP, Hershman JM, et al $\square$. The aging thyroid: Thyroid deficiency in the Framingham study. Arch Intern Med 1985;145:1386-88.

15. Bonar BD, McColgan B, Smith DF, etal. Hypothyroidism and aging: the Rosses' survey. Thyroid 2000;10: 821-7. 